\title{
Chloroplast DNA polymorphism and evolutional relationships between Asian cultivated rice (Oryza sativa) and its wild relatives (O. rufipogon)
}

\author{
W.J. Li, B. Zhang, G.W. Huang, G.P. Kang, M.Z. Liang and L.B. Chen \\ College of Life Science, Hunan Normal University, Changsha, Hunan, China \\ Corresponding authors: M.Z. Liang / L.B. Chen \\ E-mail: 1962liang@163.com / liwenjia0529@163.com
}

Genet. Mol. Res. 11 (4): 4418-4431 (2012)

Received March 12, 2012

Accepted June 6, 2012

Published October 9, 2012

DOI http://dx.doi.org/10.4238/2012.October.9.8

\begin{abstract}
We analyzed chloroplast DNA (cpDNA) polymorphism and phylogenic relationships between 6 typical indica rice, 4 japonica rice, 8 javanica rice, and 12 Asian common wild rice (Oryza rufipogon) strains collected from different latitudes in China by comparing polymorphism at 9 highly variable regions. One hundred and forty-four polymorphic bases were detected. The O. rufipogon samples had 117 polymorphic bases, showing rich genetic diversity. One hundred and thirty-one bases at 13 sites were identified with indicaljaponica characteristics; they showed differences between the indica and japonica subspecies at these sites. The javanica strains and japonica shared similar bases at these 131 polymorphic sites, suggesting that javanica is closely related to japonica. On the basis of length analyses of the open reading frame (ORF)100 and (ORF)29tRNA-Cys(GCA) ( $\left.\mathrm{TrnC}^{\mathrm{GCA}}\right)$ fragments, the O. rufipogon strains were classified into indica/japonica subgroups, which was consistent with the results of the phylogenic tree assay based on concatenated datasets. These results indicated that differences in indica and japonica also exist in the cpDNA genome of the O. rufipogon strains. However,
\end{abstract}


these differences demonstrated a certain degree of primitiveness and incompleteness, as an O. rufipogon line may show different indical japonica attributes at different sites. Consequently, O. rufipogon cannot be simply classified into the indicaljaponica types as $O$. sativa. Our data support the hypothesis that Asian cultivated rice, $O$. indica and $O$. japonica, separately evolved from Asian common wild rice (O. rufipogon) strains, which have different indica-japonica differentiation trends.

Key words: Genetic diversity; Evolutionary relationship; Dual origin; Chloroplast DNA; Indica-japonica differentiation

\section{INTRODUCTION}

Asian cultivated rice (Oryza sativa L.) is a major source of nutrition for more than half of the global population (Vaughan et al., 2003). Asian common wild rice $(O$. rufipogon Griff.) has long been believed to be the direct ancestor of Asian cultivated rice. It serves as a valuable gene pool for the improvement of cultivated rice due to its many extraordinary traits, such as disease and insect resistance, stress tolerance, cytoplasmic male sterility, and others (Khush, 1997). Therefore, a better understanding of the genetic diversity and phylogenic relationships between Asian cultivated rice and Asian common wild rice will be beneficial for the efficient use of the Oryza gene pool, and will certainly have a significant impact on rice production (Sun et al., 2001; Vaughan et al., 2003; Garris et al., 2005).

In the past decades, various phylogenetic tools have been used to investigate the evolutionary relationships between closely related Oryza species, such as morphological characteristics (Morishima and Oka, 1960), isoenzymes (Cai and Wang, 1992; Wang et al., 1994), rDNA (Fukui et al., 1994), random amplified polymorphic DNA (Sun et al., 1995), restriction fragment length polymorphism (Wang and Tanksley, 1989; Nakano et al., 1992; Sun et al., 1997, 2001), simple sequence repeats (Nishikawa et al., 2005), inter-simple sequence repeats (Joshi et al., 2000), nuclear coding sequences (Chen et al., 1994; Sun et al., 1997), single nucleotide polymorphism (SNP; Caicedo et al., 2007), and combined chloroplast, mitochondrial, and nuclear data analyses (Duan et al., 2007). Nevertheless, due to the differences in methodologies and resulting data, some reports suggested that the indicajaponica differentiation occurred not only in cultivated rice but also in its wild relatives, which provided theoretical support for the dual origin of cultivated rice. This hypothesis states that the indica and japonica subspecies separately evolved from O. rufipogon. On the other hand, other studies supported the monophyletic origin of cultivated rice, which indicates that indica descended first from O. rufipogon, and the japonica subspecies in turn evolved from indica. Hence, the genetic diversity and evolutionary relationships between $O$. sativa and $O$. rufipogon remain controversial.

The DNA of organelles has been used in phylogenetic studies due to its maternal inheritance, higher genetic stability, and lower mutation frequencies compared with nuclear DNA. In rice, the evolutionary rate of chloroplast DNA (cpDNA) is 3 times higher than that of mitochondrial DNA (mtDNA; Tian et al., 2006). Hence, its maternal inheri- 
tance and relatively high mutation rate are useful in elucidating the phylogeny of the species. Hirai et al. (1985) constructed the first physical map of rice cpDNA. Hiratsuka et al. (1989) published the rice chloroplast genome sequence from Nipponbare. Five chloroplast genome sequences of rice are available at present. In addition, open reading frame (ORF)100 has been reported as an efficient marker that determines whether a rice chloroplast genome belongs to either the indica or the japonica type. The ORF100 band of japonica rice lags behind that of indica rice as a result of a 69-bp deletion in the indica ORF100 region (Kanno et al., 1993). Chen et al. (1994) classified 137 cultivated rice into indica/japonica based on the ORF100 marker, which was consistent with the classification based on morphological and physiological characteristics. Using this marker, 151 strains of $O$. rufipogon were identified, and the cpDNA of $O$. rufipogon can also be classified into the indica and japonica types (Sun et al., 1997). Tang et al. (2004) found that indica has a 32-bp insertion in the ORF29-tRNA-Cys(GCA) ( $\mathrm{TrnC}^{\mathrm{GCA}}$ ) spacer, leading to a lag of the indica bands related to those of japonica. Therefore, ORF29-TrnCGCA can be used as another marker to distinguish the indica chloroplast genome from that of japonica. Shaw et al. (2005) found that the intron of the s16 (rps16) ribosomal protein and the spacer of TrnT ${ }^{\text {UGU-TrnL }}{ }^{\text {UAA }}$ [tRNA-Thr(UGU)-tRNA-Leu(UAA)] are highly variable regions in plant chloroplast genomes. These studies primarily focused on the sequences of the non-coding regions of cpDNA. However, some variable encoding regions of cpDNA that contain important genes involved in photosynthesis also provide effective sources for analyzing the phylogenetic relationships of closely related species.

In the present study, we performed a more comprehensive comparison of the cpDNAs of $O$. sativa and $O$. rufipogon using the sequences of 3 non-coding regions and 6 coding regions to explore the genetic diversity of cpDNA and the evolutionary relationship between $O$. sativa and O. rufipogon as well as to reveal the indica/japonica attributes of the javanica cpDNA. The results will deepen our understanding of the evolutionary relationships between $O$. sativa and $O$. rufipogon and ultimately accelerate the efficient use of the extraordinary genes of $O$. rufipogon for the genetic improvement of cultivated rice.

\section{MATERIAL AND METHODS}

\section{Comparison of chloroplast genomes}

Four chloroplast genomic sequences, O. sativa japonica (Nipponbare, AY522330.1, Tang et al., 2004 and PA64S, AY522331.1, Tang et al., 2004), O. sativa indica (isolate 9311; AY522329.1, Tang et al., 2004), and O. nivara (AP006728.1, Shahid et al., 2004), from the National Center for Biotechnology Information (NCBI) nucleotide database were compared in silico. These sequences were aligned with ClustalW, and we manually proofed the alignment for extracting encoding regions that contain many variant sites.

\section{Plant materials}

A total of 30 accessions of rice were used in this study, which contained 18 O. sativa (6 indica, 4 japonica, and 8 javanica) and 12 O. rufipogon from different latitude distributions in China (Table 1). 


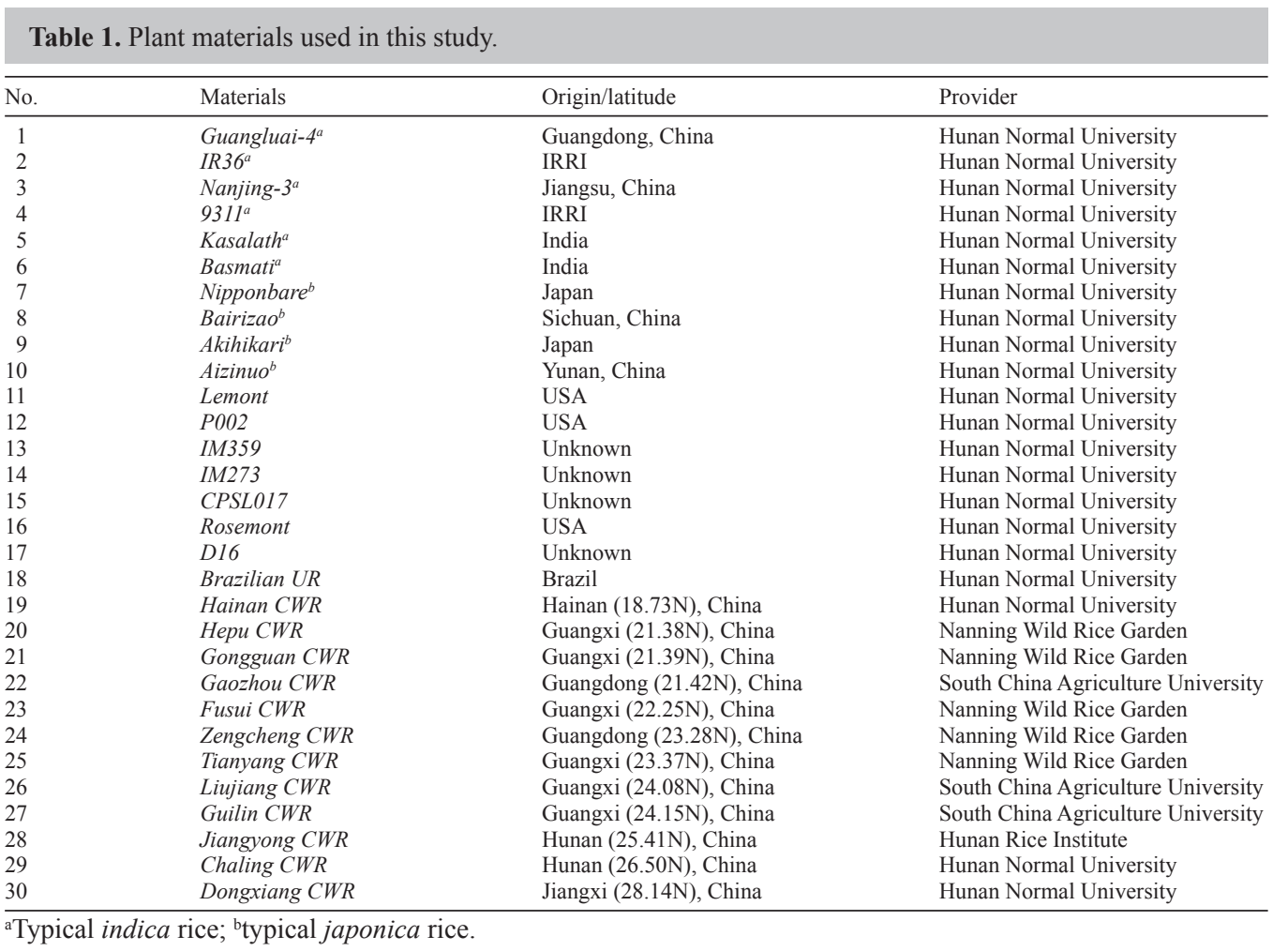

\section{Chloroplast DNA extraction}

Chloroplast DNA was extracted from the fresh leaves of rice using the method mentioned by Rogers and Bendich (1988).

\section{PCR amplification and agarose-gel electrophoresis}

Chloroplast genome primers cp1-cp11 were designed with Primer Premier 5.0 based on the chloroplast genome sequence of 9311 (Table 2). The PCR system included $2.5 \mu \mathrm{L} 10 \mathrm{X}$ PCR buffer, $0.5 \mu \mathrm{L}$ forward and reverse primers, $0.3 \mu \mathrm{L} 10 \mathrm{mM}$ dNTPs, $1 \mathrm{U}$ Taq polymerase, $40 \mathrm{ng}$ template DNA, and complementary ultrapure water to $25 \mu \mathrm{L}$. PCR amplification was performed as follows: pre-denaturation at $95^{\circ} \mathrm{C}$ for $3 \mathrm{~min}$, then $95^{\circ} \mathrm{C}$ for $30 \mathrm{~s}, 50^{\circ}-55^{\circ} \mathrm{C}$ for $30 \mathrm{~s}$, and $72^{\circ} \mathrm{C}$ for $50-90 \mathrm{~s}$; the cycle was repeated 30 times, and then $72^{\circ} \mathrm{C}$ for $10 \mathrm{~min}$. The PCR products were fractionated on a $1-3 \%$ agarose gel, which contained $0.5 \mu \mathrm{g} / \mathrm{mL}$ ethidium bromide, and then the gel was observed with an ultraviolet gel imaging system.

\section{DNA sequencing and data analysis}

The target fragments were isolated from the agarose gel, purified with the reagent 
kit [Tiangen Minipurification kit, Tiangen Biotech (Beijing) Co., Ltd.], and then directly sequenced. Sequence analyses on these polymorphic fragments were performed by the molecular evolutionary genetics analysis (MEGA) version 4.0 software (Tamura et al., 2007). GC content, consistency index, retention index, and Tajima's D statistic were calculated by MEGA 4.0. Phylogenetic analyses were conducted using maximum parsimony (MP) and neighbor joining (NJ) as implemented in MEGA 4. All positions containing gaps and missing data were eliminated from the dataset (complete deletion option). The MP analysis was carried out with all SNPs, inserts, and deletions (indels) data. The MP tree was obtained using the close-neighbor-interchange algorithm (Nei and Kumar, 2000). In the NJ analysis (Saitou and Nei, 1987), evolutionary distances were calculated using the maximum composite likelihood model as the number of base substitutions per site. Bootstrap resampling with 1000 replicates was performed to test the reliability of the inferred phylogenetic trees (Felsenstein, 1985).

Table 2. Primer sequence and target fragment of chloroplast DNA.

\begin{tabular}{|c|c|c|c|}
\hline Primer & Forward sequence $\left(5^{\prime}-3^{\prime}\right)$ & Reverse sequence $\left(5^{\prime}-3^{\prime}\right)$ & Target fragment \\
\hline сp1 & GTGGACCTGACTCCTTGAA & AGCCGAGGTCGTGGTAA & ORF100 \\
\hline $\mathrm{cp} 2$ & GCAGCCCAAGCGAGACT & AAGGCTCGGCGATACTG & ORF29-Trn $C^{G C A}$ \\
\hline $\mathrm{cp} 3$ & TTTTCTCCTCATACGGCT & TAGTCTGTTCTATTCGTCCC & rps 16 gene intron \\
\hline $\mathrm{cp} 4$ & AGTGGGCTTACATAACAGAAA & ACCAAGGCTCAATACAATCA & $\operatorname{Trn} T^{U G U}-\operatorname{Trn} L^{U A A}$ \\
\hline cp5 & AGTCTTTGCCAATGCGATAA & GTTGCTGACCCATACCAC & $p s b B$ \\
\hline cp6 & CTATGTGGTATGGGTCAGC & AAAGAAGGGATGGGAGAT & $p s b B$ \\
\hline cp7 & TAGCAACACTTTTCCCACA & GCTCATTCCAATCCTCAA & rpoc 2 \\
\hline cp8 & TGAGGATTGGAATGAGCG & TTTTGGGAGATGGTGGA & rрoc2 2 \\
\hline ср9 & TCAAGCATCTCGGTTCG & CGCCTCATTAGCCCATAC & $\operatorname{ccs} A$ \\
\hline сp10 & TTCCCAGTAAGAGGGTCAA & CTTCTCACTTGTTATGGCTTG & $n d h H$ \\
\hline cp11 & AGAGGGAAGTTGTGAGCATT & CGTCTGGGTATGCGTCCT & $p s b A$ \\
\hline
\end{tabular}

\section{RESULTS}

\section{Identifying the encoding regions with condensed variant sites in Oryza cpDNA}

We manually examined the sequence alignments by comparing the 4 chloroplast genomic sequences in the NCBI nucleotide database, and found 34 SNPs and 3 indels in the encoding regions. Of these, 19 SNPs could eventually lead to changes in the amino acids (Table 3). Five highly variable encoding regions were then selected for the genetic diversity and phylogenetic relationship analyses of rice. The 5 selected regions are fragments of photosystem II 47-kDa protein ( $p s b B)$, RNA polymerase beta chain (rpoc2), cytochrome c biogenesis protein (ccsA), NADH dehydrogenase 48-kDa subunit ( $n d h H)$, and photosystem II protein D1 ( $p s b A)$ genes.

\section{CpDNA genetic diversity analysis of 9 highly variable regions}

The ORF100, ORF29-TrnC ${ }^{\mathrm{GCA}}$, rps16 intron, and TrnT ${ }^{\mathrm{UGU}}$-TrnL ${ }^{\mathrm{UAA}}$ fragments are currently the most widely used in cpDNA genetic diversity analyses. In these 4 cpDNA regions and the 5 previously mentioned selected variable coding regions, 144 polymorphic bases (bp) were detected among 30 rice accessions (Tables 4 and 5), and 117 polymorphic bases were found among the 12 strains of $O$. rufipogon, suggesting high genetic diversity in the cpDNA of various ecotypes from different distributions. 
Table 3. SNPs and Indels found in encoding regions of rice cpDNA by comparing the four whole chloroplast genomic sequences.

\begin{tabular}{|c|c|c|c|c|c|c|}
\hline \multirow[t]{2}{*}{ Position $^{\mathrm{a}}$} & \multirow[t]{2}{*}{ Gene } & \multicolumn{4}{|c|}{ Rice species } & \multirow[t]{2}{*}{ Change of amino acid } \\
\hline & & 9311 & O. nivara & Nipponbare-G & $P A 64 S$ & \\
\hline 412 & psbA & $\mathrm{CTC}$ & CTC & TTC & TTC & Leu/Phe \\
\hline 4,547 & rps16 & TGT & TGT & GGT & GGT & Cys/Gly \\
\hline 7,141 & psbK & $\mathrm{AAC}$ & $\mathrm{AAC}$ & AAT & AAT & \\
\hline 8,554 & ORF100 & D-69 & D-69 & $\mathrm{I}-69$ & $\mathrm{I}-69$ & \\
\hline 14,166 & ORF91 & $\mathrm{AGC}$ & AGC & $\mathrm{AAC}$ & $\mathrm{AAC}$ & Ser/Asn \\
\hline 16,613 & psbM & A & - & - & - & \\
\hline 20,547 & rpoB & $\mathrm{CCA}$ & $\mathrm{CCA}$ & CCT & CCT & \\
\hline $27,979-27,980$ & rpoc2 & TTG & TTT & TGG & TGG & Leu/Phe/Trp \\
\hline 28,970 & rpoc2 & CGA & CGT & CGA & CGA & \\
\hline 29,073 & rpoc2 & $\mathrm{GAC}$ & GAC & $\mathrm{AAC}$ & $\mathrm{AAC}$ & Asp/Asn \\
\hline 29,945 & rps2 & CAA & CTA & CAA & $\mathrm{CAA}$ & Gln/Leu \\
\hline 30,925 & atpI & AAA & AAT & AAA & AAA & Lys/Asn \\
\hline 35,342 & atpA & GCA & GCA & GCG & GCG & \\
\hline 37,963 & psaB & GTC & GTT & GTC & GTC & \\
\hline 52,090 & atpB & TGC & CGC & CGC & CGC & Cys/Arg \\
\hline 53,188 & atpB & ACG & CCG & ACG & ACG & Thr/Pro \\
\hline 54,869 & $\mathrm{rbcL}$ & GGA & GGA & GGG & GGG & \\
\hline 56,545 & ORF106 & $\mathrm{TCC}$ & TCT & TCT & TCT & \\
\hline 56,818 & ORF106 & GGT & GGT & GGC & GGC & \\
\hline 63,618 & petE & CTC & TTC & CTC & CTC & Leu/Phe \\
\hline 65,172 & rpl33 & GTT & GTC & GTT & GTT & \\
\hline 65,235 & rpl33 & GCA & GCG & GCA & GCA & \\
\hline 66,354 & rpl20 & TGG & TGG & TGA & TGA & Trp/Stop condon \\
\hline 69,301 & psbB & GTG & GCG & GCG & GCG & $\mathrm{Val} / \mathrm{Ala}$ \\
\hline 69,833 & psbB & ACT & $\mathrm{ACC}$ & ACT & $\mathrm{ACT}$ & \\
\hline 76,656 & rps8 & D-21 & $\mathrm{I}-21$ & D-21 & D-21 & \\
\hline 77,532 & rpl14 & $\mathrm{ACT}$ & GCT & $\mathrm{ACT}$ & $\mathrm{ACT}$ & Thr/Ala \\
\hline 77,750 & rpl16 & TAC & TAC & TGC & TGC & Tyr/Cys \\
\hline 79,426 & rps3 & TTC & TTC & CTC & CTC & Phe/Leu \\
\hline 102,887 & ndhf & TGC & TGC & $\mathrm{CGC}$ & CGC & Cys/Arg \\
\hline 104,485 & rpl32 & ACAA & ACAA & - & - & \\
\hline 105,740 & $\operatorname{ccs} \mathrm{A}$ & TTG & TTG & TTA & TTA & \\
\hline $105,741-105,743$ & $\operatorname{ccs} \mathrm{A}$ & CTT & CTT & $\mathrm{AGC}$ & AGC & Leu/Ser \\
\hline 112,788 & ndhH & $\mathrm{ACC}$ & $\mathrm{CCC}$ & $\mathrm{ACC}$ & $\mathrm{ACC}$ & Thr/Pro \\
\hline 113,049 & ndhH & TGA & TGA & GGA & GGA & Stop condon/Gly \\
\hline
\end{tabular}

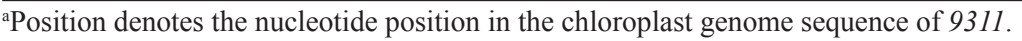

A total of 141 polymorphic bases were found in the cpDNA fragments of $O$. sativa, of which 131 indica-japonica-characteristic bases spread across 13 sites were identified (marked "4" in Tables 4 and 5). Six indica and 4 japonica strains were clearly identified at these indica-japonicaspecific sites, and 8 javanica strains had sequences similar to those of japonica at these sites. Based on the 131 indica-japonica-characteristic bases, the cpDNA classification results of these $O$. sativa strains coincide with the results of the length polymorphism of the ORF100 and ORF29-TrnC ${ }^{\mathrm{GCA}}$ fragments (Figure 1). Hence, the ORF100 and ORF29-TrnC ${ }^{\mathrm{GCA}}$ fragments are reliable molecular markers that can be used to classify the $O$. sativa cpDNA into the indica/japonica types. The $O$. rufipogon strains can also be classified into the indica/japonica types according to these 2 fragments, which agree with the results of Sun et al. (1997). Nevertheless, no O. rufipogon strain had sequences similar to those of indica or japonica in all the 131 indica-japonica-characteristic bases. Some O. rufipogon strains had more indica-specific bases, whereas the others contained more japonica-specific bases. For example, O. rufipogon from Zengcheng had 117 indica-specific bases and 14 japonicaspecific bases, whereas Chaling had 9 indica-specific bases and 122 japonica-specific bases. Therefore, although a certain indica-japonica differentiation trend exists in O. rufipogon, they could not be classified into the indicaljaponica types as simply as those in $O$. sativa. 
Table 4. Sequence divergence of the 4 cpDNA regions among 30 materials.

\begin{tabular}{|c|c|c|c|c|c|c|c|c|c|c|c|c|c|c|}
\hline \multirow[t]{3}{*}{ Material } & \multicolumn{14}{|c|}{ Gene and its base site No. } \\
\hline & \multirow{2}{*}{$\frac{\text { ORF } 100}{248-316^{a}}$} & \multicolumn{2}{|c|}{ ORF29-TrnC ${ }^{\mathrm{GCA}}$} & \multicolumn{2}{|c|}{ rps16 intron } & \multicolumn{9}{|c|}{$\operatorname{Trn} T^{\text {UGU }}-\operatorname{TrnL}^{\mathrm{UAA}}$} \\
\hline & & $130-131^{\mathrm{a}}$ & $163-194^{\mathrm{a}}$ & $267-273^{\mathrm{a}}$ & $309-312$ & $322-326^{\mathrm{a}}$ & $413^{\mathrm{a}}$ & 463 & 479 & 520 & 572 & 599 & 635 & $774-779^{\mathrm{a}}$ \\
\hline 9311 & D-69 & AA & $\mathrm{I}-32$ & CTTTATC & - & - & - & G & A & A & G & $\mathrm{T}$ & A & - \\
\hline IR36 & D-69 & AA & $\mathrm{I}-32$ & CTTTATC & - & - & - & G & A & A & G & $\mathrm{T}$ & A & - \\
\hline Nanjing-3 & D-69 & AA & $\mathrm{I}-32$ & CTTTATC & - & - & - & G & $\mathrm{C}$ & G & A & $\mathrm{T}$ & A & - \\
\hline Guangluai-4 & D-69 & AA & $\mathrm{I}-32$ & CTTTATC & CTTT & - & - & G & A & A & G & $\mathrm{T}$ & A & - \\
\hline Kasalath & D-69 & AA & $\mathrm{I}-32$ & CTTTATC & СТTT & - & - & G & A & A & G & $\mathrm{T}$ & A & - \\
\hline Basmati & D-69 & AA & $\mathrm{I}-32$ & CTTTATC & СТTT & - & - & G & A & A & G & $\mathrm{T}$ & A & - \\
\hline Nipponbare & $\mathrm{I}-69$ & GG & D-32 & - & - & TATAT & $\mathrm{T}$ & G & A & A & G & $\mathrm{T}$ & A & AGAAAA \\
\hline Bairizao & I-69 & GG & D-32 & - & - & TATAT & $\mathrm{T}$ & G & A & A & G & $\mathrm{T}$ & A & AGAAAA \\
\hline Akihikari & I-69 & GG & D-32 & - & - & TATAT & $\mathrm{T}$ & A & A & A & G & $\mathrm{C}$ & $\mathrm{T}$ & AGAAAA \\
\hline Аіzіпио & I-69 & GG & D-32 & - & - & TATAT & $\mathrm{T}$ & G & A & A & G & $\mathrm{T}$ & A & AGAAAA \\
\hline Lemont & I-69 & GG & D-32 & - & - & TATAT & $\mathrm{T}$ & G & A & A & G & $\mathrm{T}$ & A & AGAAAA \\
\hline P002 & I-69 & GG & D-32 & - & - & TATAT & $\mathrm{T}$ & G & A & A & G & $\mathrm{T}$ & A & AGAAAA \\
\hline IM359 & I-69 & GG & D-32 & - & - & TATAT & $\mathrm{T}$ & G & A & A & G & $\mathrm{T}$ & A & AGAAAA \\
\hline IM273 & I-69 & GG & D-32 & - & - & TATAT & $\mathrm{T}$ & G & A & A & G & $\mathrm{T}$ & A & AGAAAA \\
\hline CPSL017 & I-69 & GG & D-32 & - & - & TATAT & $\mathrm{T}$ & G & A & A & G & $\mathrm{T}$ & A & AGAAAA \\
\hline Rosemont & I-69 & GG & D-32 & - & - & TATAT & $\mathrm{T}$ & G & A & A & G & $\mathrm{T}$ & A & AGAAAA \\
\hline D16 & I-69 & GG & D-32 & - & - & TATAT & $\mathrm{T}$ & G & A & A & G & $\mathrm{T}$ & A & AGAAAA \\
\hline Brazilian UR & I-69 & GG & D-32 & - & - & TATAT & $\mathrm{T}$ & G & A & A & G & $\mathrm{T}$ & A & AGAAAA \\
\hline Hainan $C W R$ & D-69 & AA & $\mathrm{I}-32$ & - & - & - & - & G & A & A & G & $\mathrm{T}$ & A & AGAAAA \\
\hline Hери CWR & D-69 & AA & $\mathrm{I}-32$ & - & - & - & - & G & A & A & G & $\mathrm{T}$ & A & AGAAAA \\
\hline Gongguan CWR & D-69 & AA & $\mathrm{I}-32$ & - & - & - & - & G & A & A & G & $\mathrm{T}$ & A & AGAAAA \\
\hline Gaozhou CWR & $\mathrm{I}-69$ & GG & D-32 & - & - & - & - & G & A & A & G & $\mathrm{T}$ & A & AGAAAA \\
\hline Fusui CWR & $\mathrm{I}-69$ & GG & D-32 & - & - & - & - & G & A & A & G & $\mathrm{T}$ & A & AGAAAA \\
\hline Zengcheng $C W R$ & D-69 & AA & $\mathrm{I}-32$ & - & - & - & - & G & A & A & G & $\mathrm{T}$ & A & AGAAAA \\
\hline Tianyang $C W R$ & D-69 & AA & $\mathrm{I}-32$ & - & - & - & - & G & A & A & G & $\mathrm{T}$ & A & AGAAAA \\
\hline Liujiang $C W R$ & $\mathrm{I}-69$ & GG & D-32 & - & - & TATAT & $\mathrm{T}$ & G & A & A & G & $\mathrm{T}$ & A & AGAAAA \\
\hline Guilin CWR & $\mathrm{I}-69$ & GG & D-32 & - & - & TATAT & $\mathrm{T}$ & G & A & A & G & $\mathrm{T}$ & A & AGAAAA \\
\hline Jiangyong $C W R$ & D-69 & AA & $\mathrm{I}-32$ & - & - & - & - & G & A & A & G & $\mathrm{T}$ & A & AGAAAA \\
\hline Chaling CWR & I-69 & GG & D-32 & - & - & TATAT & $\mathrm{T}$ & G & A & A & G & $\mathrm{T}$ & A & AGAAAA \\
\hline Dongxiang $C W R$ & D-69 & AA & $\mathrm{I}-32$ & - & - & - & - & $\mathrm{G}$ & A & A & G & $\mathrm{T}$ & A & AGAAAA \\
\hline
\end{tabular}

aindica-japonica-characteristic sites. All the GenBank accession Nos. of these sequences were listed in Table S1.

\section{Sequence characteristics and phylogenetic analysis based on concatenated datasets}

A total of 9080 bases of the cpDNA regions were sequenced separately from 30 Oryza accessions. The variable sites, varying from $1(0.15 \%)$ to $6(0.73 \%)$, were screened in all of the sampled sequences, except in the ORF100 region. The ORF100, ORF29TrnC ${ }^{\mathrm{GCA}}$, rps16 intron, and $\mathrm{TrnT}^{\mathrm{UGU}}-\mathrm{TrnL}^{\mathrm{UAA}}$ regions contained different numbers of informative indels, varying from 12 to $69 \mathrm{bp}$ (Table 6). The total number of variable sites was $22(0.24 \%)$, and that of the parsimoniously informative characters was $14(0.15 \%)$. We tested the selection and neutrality with Tajima's D, using the sequences of $O$. sativa and O. rufipogon in each region. The results showed no significant value in any of the regions, which indicated that the areas are evolutionarily neutral and without selective pressure.

Two phylogenetic trees were reconstructed via the MP and NJ methods using the combined sequences of the 9 cpDNA regions to reveal the evolutionary relationship between $O$. sativa and $O$. rufipogon. The MP phylogenetic tree was assembled from all of the SNPs 
(22 sites) and the length variations (8 sites) to fully extract information regarding the DNA sequences (Figure 2). On the other hand, the NJ tree was constructed based only on the SNP data (Figure 3). The MP and NJ trees showed nearly similar topologies, with little difference in the bootstrap values, indicating that the studied rice species were classified into 2 main clusters: 1 branch consisted of the indica and the 8 O. rufipogon strains, and the other consisted of the japonica, javanica, and the rest of the 4 O. rufipogon strains. These relationships were supported by bootstrap values of greater than $50 \%$ (Figures 2 and 3). The results also support the existence of indica-japonica differentiation trends in O. rufipogon.

Table 5. Sequence divergence of the 5 new selected cpDNA encoding regions among 30 materials.

\begin{tabular}{|c|c|c|c|c|c|c|c|c|}
\hline \multirow[t]{3}{*}{ Material } & \multicolumn{7}{|c|}{ Gene and its base site No. } & \multirow{3}{*}{$\begin{array}{r}p s b A \\
331^{\mathrm{a}}\end{array}$} \\
\hline & \multicolumn{2}{|c|}{$p s b B$} & \multicolumn{2}{|c|}{ rpoc2 } & \multirow{2}{*}{$\frac{c \operatorname{cs} A}{540-543^{\mathrm{a}}}$} & \multicolumn{2}{|c|}{$n d h H$} & \\
\hline & $550^{\mathrm{a}}$ & 1082 & $3293^{\mathrm{a}}, 3294$ & $4387^{\mathrm{a}}$ & & 109 & $370^{\mathrm{a}}$ & \\
\hline 9311 & $\mathrm{~T}$ & $\mathrm{~T}$ & TG & $\mathrm{G}$ & GCTT & $\mathrm{A}$ & $\mathrm{T}$ & $\mathrm{C}$ \\
\hline IR36 & $\mathrm{T}$ & $\mathrm{T}$ & TG & G & GCTT & $\mathrm{A}$ & $\mathrm{T}$ & $\mathrm{C}$ \\
\hline Nanjing-3 & $\mathrm{T}$ & $\mathrm{T}$ & TG & G & GCTT & A & $\mathrm{T}$ & $\mathrm{C}$ \\
\hline Guangluai-4 & $\mathrm{T}$ & $\mathrm{T}$ & TG & G & GCTT & A & $\mathrm{T}$ & $\mathrm{C}$ \\
\hline Kasalath & $\mathrm{T}$ & $\mathrm{T}$ & TG & $\mathrm{G}$ & GCTT & $\mathrm{A}$ & $\mathrm{T}$ & $\mathrm{C}$ \\
\hline Basmati & $\mathrm{T}$ & $\mathrm{T}$ & TG & G & GCTT & A & $\mathrm{T}$ & $\mathrm{C}$ \\
\hline Nipponbare & $\mathrm{C}$ & $\mathrm{T}$ & GG & A & AAGC & $\mathrm{A}$ & $\mathrm{G}$ & $\mathrm{T}$ \\
\hline Bairizao & $\mathrm{C}$ & $\mathrm{T}$ & GG & A & AAGC & A & G & $\mathrm{T}$ \\
\hline Akihikari & $\mathrm{C}$ & $\mathrm{T}$ & GG & A & AAGC & A & G & $\mathrm{T}$ \\
\hline Aizinuo & $\mathrm{C}$ & $\mathrm{T}$ & GG & $\mathrm{A}$ & AAGC & A & G & $\mathrm{T}$ \\
\hline Lemont & $\mathrm{C}$ & $\mathrm{T}$ & GG & A & AAGC & A & G & $\mathrm{T}$ \\
\hline P002 & $\mathrm{C}$ & $\mathrm{T}$ & GG & A & AAGC & A & G & $\mathrm{T}$ \\
\hline IM359 & $\mathrm{C}$ & $\mathrm{T}$ & GG & A & AAGC & A & G & $\mathrm{T}$ \\
\hline IM273 & $\mathrm{C}$ & $\mathrm{T}$ & GG & $\mathrm{A}$ & AAGC & $\mathrm{A}$ & G & $\mathrm{T}$ \\
\hline CPSL017 & $\mathrm{C}$ & $\mathrm{T}$ & GG & A & AAGC & A & $\mathrm{G}$ & $\mathrm{T}$ \\
\hline Rosemont & $\mathrm{C}$ & $\mathrm{T}$ & GG & $\mathrm{A}$ & AAGC & $\mathrm{A}$ & $\mathrm{G}$ & $\mathrm{T}$ \\
\hline D16 & $\mathrm{C}$ & $\mathrm{T}$ & GG & A & AAGC & A & G & $\mathrm{T}$ \\
\hline Brazilian UR & $\mathrm{C}$ & $\mathrm{T}$ & GG & $\mathrm{A}$ & AAGC & $\mathrm{A}$ & G & $\mathrm{T}$ \\
\hline Hainan $C W R$ & $\mathrm{C}$ & $\mathrm{T}$ & TG & G & GCTT & A & $\mathrm{T}$ & $\mathrm{C}$ \\
\hline Hери CWR & $\mathrm{T}$ & $\mathrm{T}$ & TG & $\mathrm{G}$ & GCTT & $\mathrm{A}$ & $\mathrm{T}$ & $\mathrm{C}$ \\
\hline Gongguan CWR & $\mathrm{T}$ & $\mathrm{T}$ & GG & $\mathrm{G}$ & GCTT & $\mathrm{A}$ & $\mathrm{T}$ & $\mathrm{C}$ \\
\hline Gaozhou CWR & $\mathrm{C}$ & $\mathrm{T}$ & GG & A & GCTT & A & G & $\mathrm{T}$ \\
\hline Fusui CWR & $\mathrm{C}$ & $\mathrm{T}$ & GG & $\mathrm{A}$ & GCTT & $\mathrm{A}$ & $\mathrm{G}$ & $\mathrm{T}$ \\
\hline Zengcheng CWR & $\mathrm{C}$ & $\mathrm{T}$ & TT & $\mathrm{G}$ & GCTT & A & $\mathrm{T}$ & $\mathrm{C}$ \\
\hline Tianyang $C W R$ & $\mathrm{C}$ & $\mathrm{C}$ & TT & G & GCTT & $\mathrm{C}$ & $\mathrm{T}$ & $\mathrm{C}$ \\
\hline Liujiang $C W R$ & $\mathrm{C}$ & $\mathrm{T}$ & GG & A & GCTT & A & $\mathrm{G}$ & $\mathrm{T}$ \\
\hline Guilin CWR & $\mathrm{C}$ & $\mathrm{T}$ & GG & G & GCTT & A & G & $\mathrm{T}$ \\
\hline Jiangyong CWR & $\mathrm{T}$ & $\mathrm{T}$ & TG & A & GCTT & A & $\mathrm{G}$ & $\mathrm{T}$ \\
\hline Chaling $\stackrel{C}{C} W R$ & $\mathrm{~T}$ & $\mathrm{~T}$ & TG & G & GCTT & A & $\mathrm{T}$ & $\mathrm{C}$ \\
\hline Dongxiang $C W R$ & $\mathrm{~T}$ & $\mathrm{~T}$ & TG & G & GCTT & A & $\mathrm{T}$ & $\mathrm{C}$ \\
\hline
\end{tabular}

aindica-japonica-characteristic sites. All the GenBank accession Nos. of these sequences were listed in Table S1.

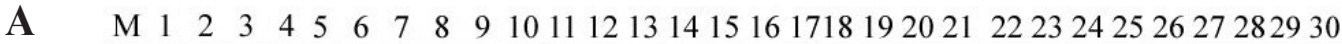

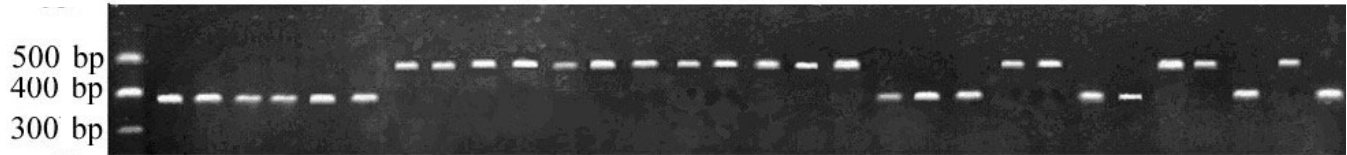

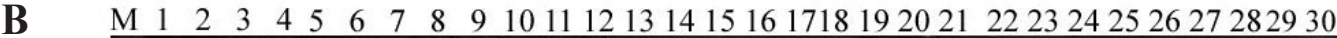

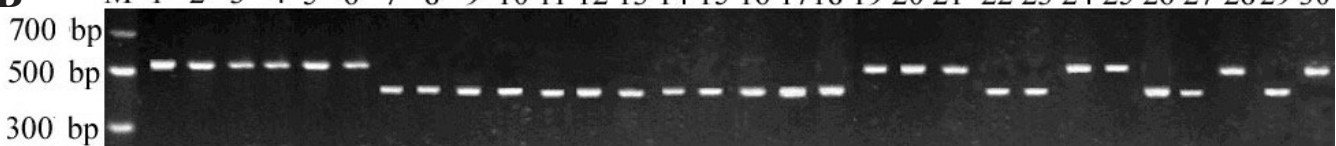

Figure 1. Agarose gel eletrophoresis results of ORF100 and ORF29-TrnC $\mathrm{CCA}^{\mathrm{GCA}}$ fragments. A. ORF100 fragments; B. ORF29-TrnC ${ }^{\mathrm{GCA}}$ fragments. Lane $M=$ molecular marker; lanes 1-30= different materials according to Table 1. 
Table 6. Sequence characteristics of 9 regions and combined datasets.

\begin{tabular}{|c|c|c|c|c|c|c|c|c|}
\hline Locus & $\begin{array}{l}\text { Aligned } \\
\text { length (bp) }\end{array}$ & GC (\%) & $\begin{array}{l}\text { No. of variable } \\
\text { sites }(\%)\end{array}$ & $\begin{array}{c}\text { No. of } \\
\text { informative } \\
\text { sites (\%) }\end{array}$ & $\begin{array}{l}\text { Consistency } \\
\text { index }\end{array}$ & $\begin{array}{l}\text { Retention } \\
\text { index }\end{array}$ & $\begin{array}{c}\text { No. of } \\
\text { informative } \\
\text { indels }\end{array}$ & Tajima's D \\
\hline ORF100 & 456 & 32.5 & 0 & 0 & - & - & $1(69 \mathrm{bp})$ & $\mathrm{n} / \mathrm{c}$ \\
\hline ORF29-TrnC $\mathrm{C}^{\mathrm{GCA}}$ & 514 & 37.8 & $2(0.39)$ & $2(0.39)$ & 1.000 & 1.000 & $1(32 b p)$ & 1.083071 \\
\hline rps16 intron & 684 & 32.1 & $1(0.15)$ & 0 & - & - & $3(17 \mathrm{bp})$ & -1.155588 \\
\hline $\operatorname{TrnT}^{\text {UGU_TrnL }} \mathrm{LAA}^{\mathrm{UAA}}$ & 824 & 29.3 & $6(0.73)$ & 0 & - & - & 3 (12 bp) & -1.091074 \\
\hline psbB & 1688 & 43.9 & $2(0.12)$ & $2(0.12)$ & 1.000 & 1.000 & 0 & 0.737635 \\
\hline rpoc2 & 2474 & 38.1 & $4(0.16)$ & $3(0.12)$ & 0.800 & 0.960 & 0 & 0.959302 \\
\hline $\operatorname{ccs} \mathrm{A}$ & 859 & 33.9 & $4(0.47)$ & $4(0.47)$ & 1.000 & 1.000 & 0 & 1.092620 \\
\hline ndhH & 964 & 37.8 & $2(0.21)$ & $2(0.21)$ & 1.000 & 1.000 & 0 & 0.838228 \\
\hline psbA & 617 & 42.9 & $1(0.16)$ & $1(0.16)$ & 1.000 & 1.000 & 0 & 1.590843 \\
\hline Total & 9080 & 37.5 & $22(0.24)$ & $14(0.15)$ & 1.000 & 1.000 & 8 (130 bp) & 0.413816 \\
\hline
\end{tabular}

$\mathrm{n} / \mathrm{c}=$ not counted.

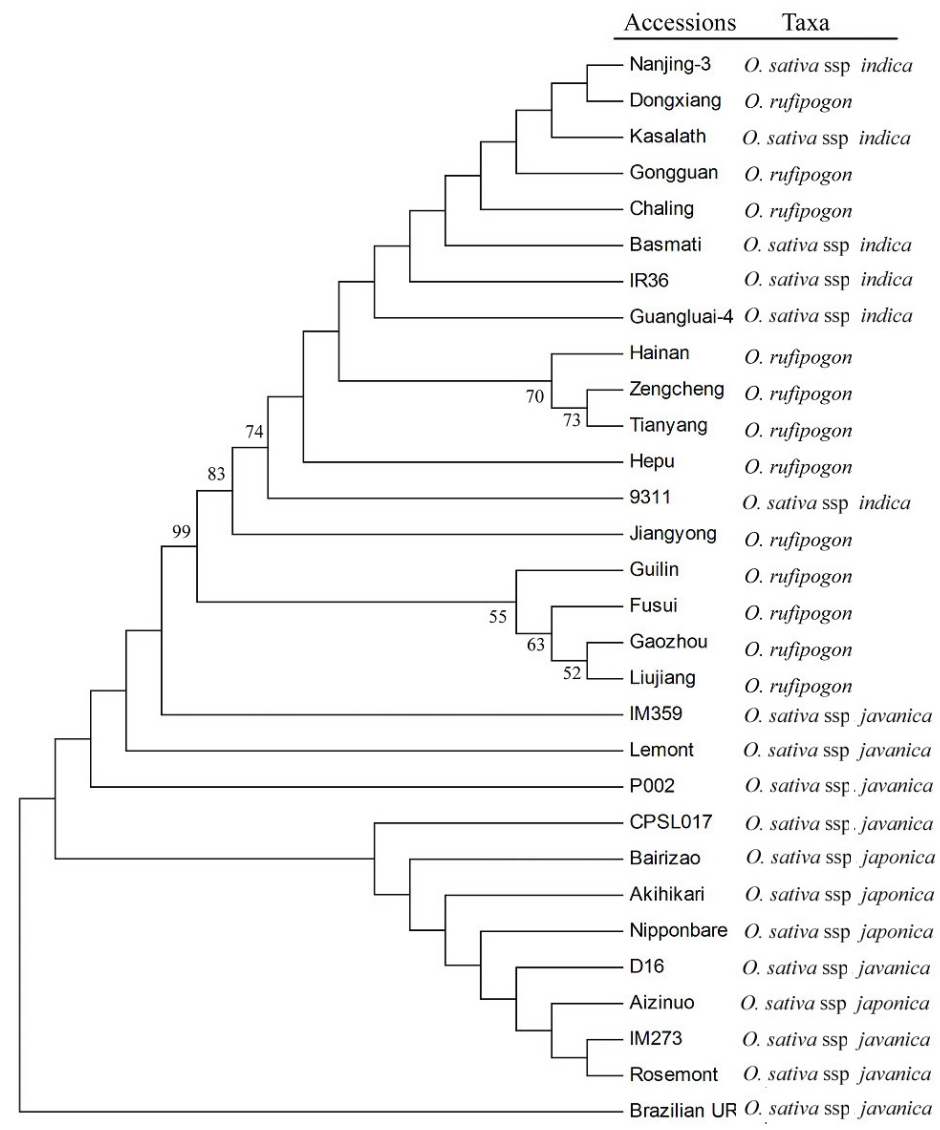

Figure 2. Evolutionary history inferred using the maximum parsimony method. A strict consensus tree of the 288 most parsimonious trees (length $=25$ ) is shown. The consistency index is 0.705882 , the retention index is 0.960938 , and the composite index is 0.768750 for all sites and parsimony-informative sites. The MP tree was obtained using the close-neighbor-interchange algorithm. The bootstrap consensus tree inferred from 1000 replicates is taken to represent the evolutionary history of the taxa analyzed. Branches corresponding to partitions reproduced in less than $50 \%$ bootstrap replicates are collapsed. The percentages of replicate trees in which the associated taxa clustered together in the bootstrap test (1000 replicates) are shown next to the branches. 


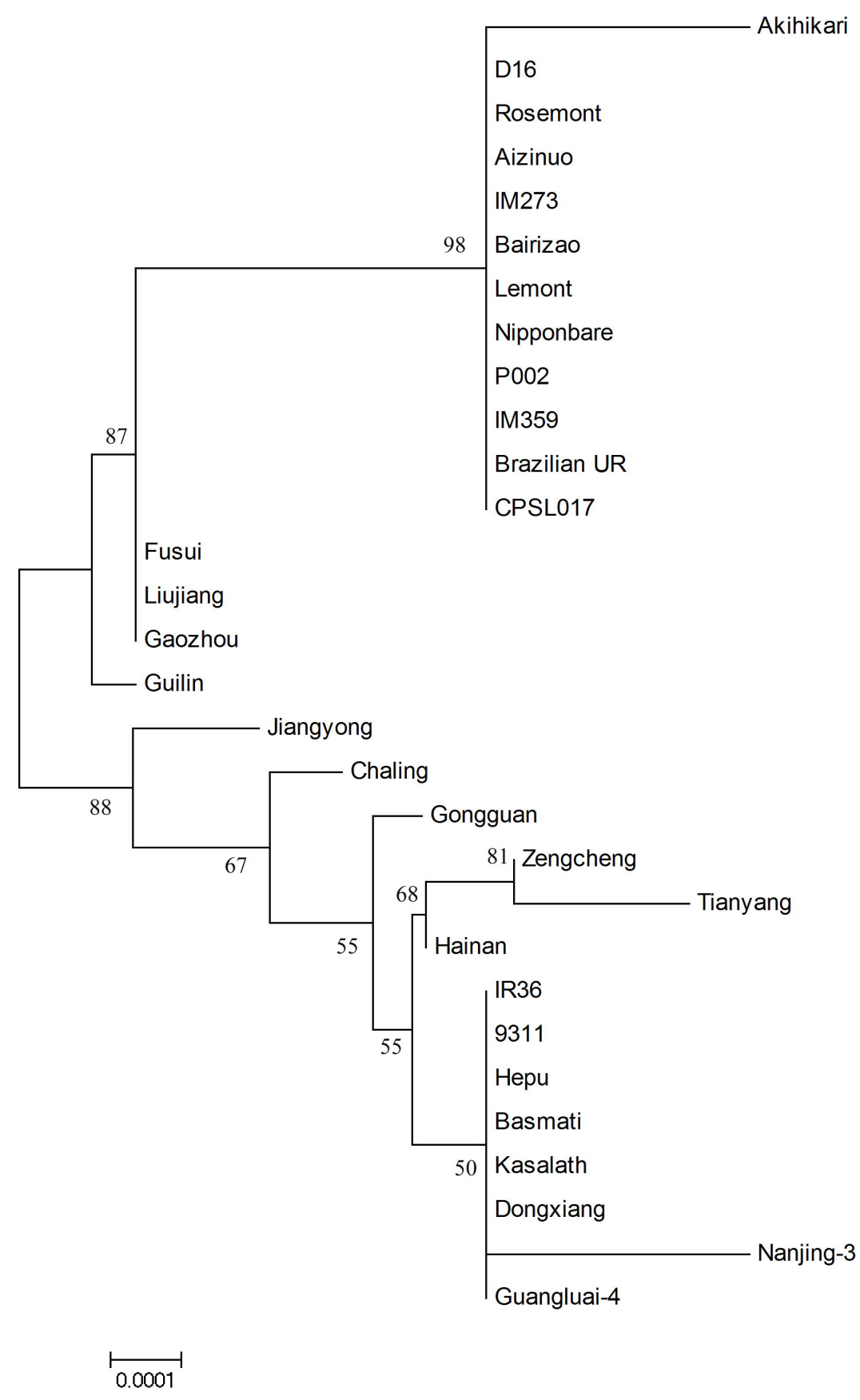

Figure 3. Evolutionary history inferred using the neighbor-joining method. The optimal tree with the sum of branch length $=0.00286400$ is shown. The evolutionary distances were computed using the maximum composite likelihood model. The percentages of replicate trees in which the associated taxa clustered together in the bootstrap test (1000 replicates) are shown next to the branches. 


\section{DISCUSSION}

\section{CpDNA genetic diversity of $O$. sativa}

Indica and japonica are 2 important subspecies of $O$. sativa. Their agronomic characteristics and ecological adaptability show remarkable differences, and this characteristic association of traits has been explained by hybrid sterility or by reproductive barriers (Sato and Morishima, 1988; Harushima et al., 1998). The nuclear genetic diversities between indica and japonica, particularly the nuclear genetic differences related to important agronomic traits, have been widely investigated in recent decades (Second, 1982; Wang and Tanksley, 1989; Chen et al., 1994; Garris et al., 2005). However, our understanding of the cytoplasmic genetic diversity between them, as well as of the relationship between their genetic diversity, agronomic characteristics, and phylogeny, is still limited (Kanno et al., 1993; Chen et al., 1994; Sun et al., 1997; Shaw et al., 2005). In the present study, 131 indica-japonica characteristic bases involved in 13 sites were identified based on the sequence analysis of 6 indica, 4 japonica, and 8 javanica, as listed in Table 1. The results revealed abundant polymorphism between the indica and japonica subspecies, not only in the non-coding regions, but also in the coding regions. These polymorphic bases in the encoding regions could lead to changes in the amino acids. However, whether these amino acid changes are related to the different phenotypes of these 2 subspecies remains to be determined. The indica-japonica classification results of these $O$. sativa strains in all of the indica-japonica characteristic nucleotides are highly consistent with those of the ORF100 and ORF29-TrnC ${ }^{\mathrm{GCA}}$ fragments. Therefore, the analysis of the amplified fragment lengths of ORF100 and ORF29-TrnC ${ }^{\mathrm{GCA}}$ using agarose gel electrophoresis is a simple, fast, and accurate method of identifying the indica/japonica types in the $O$. sativa cytoplasm.

Javanica showed very wide compatibility, as shown by the fairly good bearing rates of both its indica/javanica and japonica/javanica hybrids, which has great significance for achieving strong heterosis. However, the attributes of javanica remain controversial (Wang and Sun, 2000). Some scholars advocated that $O$. sativa L. should be classified into the indica (O. sativa ssp indica) and japonica (O. sativa ssp japonica) subspecies (Cheng, 1985), and that javanica is an ecotype of japonica. However, others argued that it should be divided into 3 subspecies, namely, indica, japonica, and javanica (Chang, 1976; Yuan, 1987). The javanica studied in our research has a relatively close relationship with japonica and is clustered into the japonica branch in both the MP and NJ trees; therefore, our results support the former classification system.

\section{CpDNA genetic diversity and indica-japonica differentiation in O. rufipogon}

The O. rufipogon strains used in our study were distributed from Yaxian $(18.73 \mathrm{~N})$ of the Hainan Province to Dongxiang $(28.14 \mathrm{~N}$; the highest latitude of $O$. rufipogon distribution) of the Jiangxi Province, both located in China. The plant types, photosensitivities, cold tolerance, and overwintering capabilities of the $O$. rufipogon strains from the different latitude distributions showed clear differences. The O. rufipogon strains from Dongxiang $(28.14 \mathrm{~N})$, Chaling $(26.50 \mathrm{~N})$, Jiangyong $(25.41 \mathrm{~N})$, Guilin $(24.15 \mathrm{~N})$, and Liujiang $(24.08 \mathrm{~N})$ can tassel, bloom, and seed from September to November under natural conditions in Changsha $(28.12 \mathrm{~N})$, whereas those from Hainan $(18.73 \mathrm{~N})$ can only complete their entire life cycle under a 10-h short-day treatment. These results indicated differences in the photosensitivity of 
floral induction, although the O. rufipogon strains were all typical short-day plants, the photosensitivity strengthened with decreasing distribution latitude. These $O$. rufipogon strains also showed significant differences in their cold tolerance and overwintering capabilities under natural conditions in Changsha. Those from the lowest latitude of Hainan $(18.73 \mathrm{~N})$ could not live through winter; those from the $21.38 \mathrm{~N}$ to $25.41 \mathrm{~N}$ region could not survive overwintering in normal years without freezing weather, and only those from Chaling $(26.50 \mathrm{~N})$ and Dongxiang $(28.14 \mathrm{~N})$ safely survived the winter of 2008 , which had a rare snow disaster that lasted for 20 days. These results demonstrated the biological diversity of $O$. rufipogon, which resulted from the difference in regional distributions ( $\mathrm{Li}$ et al., 2001).

Our study also indicated rich genetic polymorphism in the cpDNA of $O$. rufipogon from different distributions. The $O$. rufipogon strains from near geographic distributions often had similar sequences and closer relationships. For instance, the O. rufipogon from Gaozhou $(21.42 \mathrm{~N})$ and Fusui $(22.25 \mathrm{~N})$ had perfectly similar sequences and were clustered into a clade based on the sequence analyses of these 9 highly cpDNA variable regions (Figures 2 and 3). The O. rufipogon strains showed different trends of indica-japonica differentiation in the 131 identified indica-japonica characteristic bases, which were validated by both the MP and NJ trees. Hence, these trends provide evidence on the level of cytoplasmic genetic information for dual origins; indica and japonica must have evolved separately from O. rufipogon strains with different indica/japonica differentiation trends (Chang, 1976; Second, 1982; Londo et al., 2006; Duan et al., 2007; Kumagai et al., 2010). However, no O. rufipogon strains that are completely identical to those of indica or japonica were found at these sites in our research, suggesting that the indica-japonica differentiation of $O$. rufipogon is relatively primitive and incomplete. Thus, the agarose gel electrophoresis results of the ORF100 and ORF29-TrnCGCA fragments could only be used in the preliminary analysis of the indica-japonica differentiation trends in $O$. rufipogon, and we could not simply determine the characteristics of the $O$. rufipogon cpDNA based on these results. In addition, all of these corresponding indicaljaponicaspecific sequences, except the indica-specific sequences "CTTTATC" at positions 267-273 of the rps 16 intron and the japonica-specific sequences "AAGC" at positions 540-543 of the $\operatorname{ccs} A$ gene, could be detected in the O. rufipogon strains. When position 370 of the $n d h H$ gene fragment is taken as an example, the indica-specific base $\mathrm{C}$ was found in O. rufipogon from Gaozhou, Fusui, Liujiang, Guilin, and Jiangyong, whereas the japonica-specific sequence G was found in the rest of the O. rufipogon strains (Tables 4 and 5). Hence, the indica/japonicaspecific sequences of the cpDNA in $O$. sativa were mainly inherited from the $O$. rufipogon strains that have different indica/japonica differentiation trends. In addition, a small number of indica/japonica-specific sequences have independently formed through mutation during the long process of natural selection and artificial selection. This phenomenon is another theory that supports the dual origin of $O$. sativa.

\section{ACKNOWLEDGMENTS}

We thank the South China Agriculture University and Nanning Wild Rice Garden for providing the plant material for this study. Research supported by the National High Technology Research and Development Program (863 Program, \#2010AA101305), Key Programs for Science and Technology Development of Hunan Province (\#2009FJ1004-1) and the National Natural Science Foundation of China (\#31171473/C1302). 


\section{Supplementary material}

\section{REFERENCES}

Cai HW and Wang XK (1992). Classification of Asian rices by esterase isozymes. Southeast China J. agric. Sci. 5: 19-22.

Caicedo AL, Williamson SH, Hernandez RD, Boyko A, et al. (2007). Genome-wide patterns of nucleotide polymorphism in domesticated rice. PLoS Genet. 3: 1745-1756.

Chang TT (1976). The origin, evolution, cultivation, dissemination, and diversification of Asian and African rices. Euphytica 25: 435-441.

Chen WB, Sato YI, Nakamura I and Nakai H (1994). Indica-Japonica differentiation in Chinese rice landraces. Euphytica 74: 195-201.

Cheng KS (1985). A statistical evaluation of the classification of rice cultivars into hsien and keng subspecies. RGN 2: 46-48.

Duan SH, Li SQ, Li YS, Xiong Y, et al. (2007). Distribution and SNPs of the rice CMS-related gene in AA-genome of Oryza species. Yi Chuan 29: 455-461.

Felsenstein J (1985). Confidence limits on phylogenies: an approach using the bootstrap. Evolution 39: 783-791.

Fukui K, Ohmido N and Khush GS (1994). Variability in rDNA loci in the genus Oryza detected through fluorescence in situ hybridization. Theor. Appl. Genet. 87: 893-899.

Garris AJ, Tai TH, Coburn J, Kresovich S, et al. (2005). Genetic structure and diversity in Oryza sativa L. Genetics 169: 1631-1638.

Harushima Y, Kurata N, Yano M, Sasaki T, et al. (1998). Detection of interactive loci for genotype segregation in F2 populations between japonica and indica rice crosses. Breed. Sci. 4: 82-101.

Hirai A, Isshibashi T, Morikami A, Iwatsuki N, et al. (1985). Rice chloroplast DNA: a physical map and the location of the genes for the large subunit of ribulose 1, 5-bisphosphate carboxylase and the $32 \mathrm{kD}$ photosystem II reaction center protein. Theor. Appl. Genet. 70: 117-122.

Hiratsuka J, Shimada H, Whittier R, Ishibashi T, et al. (1989). The complete sequence of the rice (Oryza sativa) chloroplast genome: intermolecular recombination between distinct tRNA genes accounts for a major plastid DNA inversion during the evolution of the cereals. Mol. Gen. Genet. 217: 185-194.

Joshi SP, Gupta VS, Aggarwal RK, Ranjekar PK, et al. (2000). Genetic diversity and phylogenetic relationship as revealed by inter simple sequence repeat (ISSR) polymorphism in the genus Oryza. Theor. Appl. Genet. 100: 1311-1320.

Kanno A, Watanabe N, Nakamura I and Hirai A (1993). Variations in chloroplast DNA from rice (Oryza sativa): Differences between deletions mediated by short direct-repeat sequences within a single species. Theor. Appl. Genet. 86: 579-584.

Khush GS (1997). Origin, dispersal, cultivation and variation of rice. Plant Mol. Biol. 35: 25-34.

Kumagai M, Wang L and Ueda S (2010). Genetic diversity and evolutionary relationships in genus Oryza revealed by using highly variable regions of chloroplast DNA. Gene 462: 44-51.

Li DY, Liang YM and Yang HQ (2001). Genetic diversity of agricultural crops germplams in Guangxi. Acta Bot. Yunnanica 23: 18-21.

Londo JP, Chiang YC, Hung KH, Chiang TY, et al. (2006). Phylogeography of Asian wild rice, Oryza rufipogon, reveals multiple independent domestications of cultivated rice, Oryza sativa. Proc. Natl. Acad. Sci. U. S. A. 103: 9578-9583.

Morishima $\mathrm{H}$ and Oka HI (1960). The pattern of interspecific variation in the genus Oryza: Its quantitative representation by statistical methods. Evolution 14: 153-165.

Nakano M, Yoshinura A and Iwata N (1992). Phylogenetic study of cultivated rice and its wild relatives by RFLP. Rice Genet. Newsl. 9: 132-134.

Nei M and Kumar S (2000). Molecular Evolution and Phylogenetics. Oxford University Press, New York.

Nishikawa T, Vaughan DA and Kadowaki K (2005). Phylogenetic analysis of Oryza species, based on simple sequence repeats and their flanking nucleotide sequences from the mitochondrial and chloroplast genomes. Theor. Appl. Genet. 110: 696-705.

Rogers OS and Bendich AJ (1988). Extraction of DNA Plant Tissue, Plant Molecular. In: Plant Molecular Biology Manual (Gelvin SB, Schilpe RA and Verna DS, eds.). Kluwer Academic Publishers, Dordecht, 1-10.

Saitou N and Nei M (1987). The neighbor-joining method: a new method for reconstructing phylogenetic trees. Mol. Biol. Evol. 4: 406-425.

Sato YI and Morishima H (1988). Distribution of the genes causing F2 chlorosis in rice cultivars of the Indica and Japonica types. Theor. Appl. Genet. 75: 723-727.

Second G (1982). Origin of the genic diversity of cultivated rice (Oryza spp.): study of the polymorphism scored at 40 isozyme loci. Jap. J. Genet. 57: 25-57.

Shahid MM, Nishikawa T, Fukuoka S, Njenga PK, et al. (2004). The complete nucleotide sequence of wild rice (Oryza nivara) 
chloroplast genome: first genome wide comparative sequence analysis of wild and cultivated rice. Gene 340: 133-139.

Shaw J, Lickey EB, Beck JT, Farmer SB, et al. (2005). The tortoise and the hare II: relative utility of 21 noncoding chloroplast DNA sequences for phylogenetic analysis. Am. J. Bot. 92: 142-166.

Sun CQ, Mao L, Wang ZS, Zhu LH, et al. (1995). A Primary Study of Cultivated Rice (Oryza sativa) and Common Chinese Wild Rice (O. rufipogon) Using Random Amplified Polymorphic DNA (RAPD). Chin. J. Rice Sci. 9: 1-6.

Sun CQ, Wang XK, Yoshimura A and Iwata N (1997). Indica-japonica differentiation of chloroplast DNA in O. rufipogon Griff. and O. sativa L. J. Agric. Biotech. 5: 319-324.

Sun CQ, Wang XK, Li ZC, Yoshimura A, et al. (2001). Comparison of the genetic diversity of common wild rice (Oryza rufipogon Griff.) and cultivated rice (O. sativa L.) using RFLP markers. Theor. Appl. Genet. 102: 157-162.

Tamura K, Dudley J, Nei M and Kumar S (2007). MEGA4: Molecular Evolutionary Genetics Analysis (MEGA) software version 4.0. Mol. Biol. Evol. 24: 1596-1599.

Tang J, Xia H, Cao M, Zhang X, et al. (2004). A comparison of rice chloroplast genomes. Plant Physiol. 135: 412-420.

Tian X, Zheng J, Hu S and Yu J (2006). The rice mitochondrial genomes and their variations. Plant Physiol. 140: 401-410.

Vaughan DA, Morishima H and Kadowaki K (2003). Diversity in the Oryza genus. Curr. Opin. Plant Biol. 6: 139-146.

Wang XK and Sun CQ (2000). Origin and evolution of biodiversity and classification of Oryza sativa L. J. Plant Genet. Resour. 1: 48-53.

Wang XK, Cai HW, Sun CQ, Wang ZS, et al. (1994). The preliminary study on the primitive type of Oryza rufipogon Griff. in China and its Hsien-Keng differentiation. Chin. J. Rice Sci. 8: 205-210.

Wang ZY and Tanksley SD (1989). Restriction fragment length polymorphism in Oryza sativa L. Genome 32: 1113-1118.

Yuan LP (1987). The stratagement thinking of hybrid rice breeding. Hybrid Rice 2: 1-3. 